IFJPAN-IV-2019-9

\title{
DIS with KaTie
}

\section{A. van Hameren*t}

Institute of Nuclear Physics, Polish Academy of Sciences, Kraków

E-mail: hameren@ifj.edu.pl

KaTie is a parton-level event generator that allows for the use of factorization prescriptions requiring space-like initial-state partons. Some updates are presented, in particular its application to DIS.

XXVII International Workshop on Deep-Inelastic Scattering and Related Subjects - DIS2019 8-12 April, 2019

Torino, Italy

* Speaker.

${ }^{\dagger}$ This work was supported by grant of National Science Center, Poland, No. 2015/17/B/ST2/01838. 


\section{Introduction}

Due to their complexity, calculations for multi-jet processes at particle colliders require automated computer programs. Parton-level automation was established for tree-level calculations almost two decades ago [1-5]. First of all it is the multitude of processes and Feynman graphs that necessitate automation, but also the phase space integration to obtain distributions of observables is highly non-trivial. The observables usually include phase space cuts that leave the Monte Carlo method of numerical integration as the only option. Since the amplitudes involved must then be evaluated numerically for each of the many generated phase space points, it turns out to be most efficiently performed via numerical recursion, completely avoiding large expressions.

When hadrons are involved as scattering particles, then the calculation of differential cross sections requires the factorization of the high-energy degrees of freedom of the scattering process from the low-energy degrees of freedom related to the structure of the hadrons. The former can be completely determined via perturbation theory, while the latter involves non-perturbative ingredients. Factorization is established using the parton model of hadrons, and hard scattering process is described by the partonic cross section, while the relevant structure of the hadron is encoded into parton distribution functions (PDFs).

In the well-established collinear factorization, the initial-state partons are considered to be onshell, and most automated programs are restricted to this application. When the energy fraction $x$ of the intial-state hadron passed to the initial-state parton involved in the hard scattering is small, then the momentum components $k_{T}^{\mu}$ transverse to the momentum of the intital-state hadron can often not be considered to be negligible anymore. In this picture of $k_{T}$-factorization, or high-energy factorization, the initial-state partons cannot be on-shell [6,7]. KaTie [8] is an automated partonlevel event generator that can deal with such factorization approaches. On the technical level, the most essential difference is in the amplitudes associated with the scatting processes, which now have space-like, rather than light-like, partons. They need to be defined and calculated without breaking gauge invariance, and in KaTie the method of [9] is applied. In [10] it is pointed out how QED gauge invariance - an issue the non-triviality of which was highlighted recently in [11] - is ensured too. KaTie has been used in several recent phenomenological studies [12-14]. The fact that it can produce parton-level event files with space-like initial-state partons opens the possibility to match it with a parton shower without the need to compromise the kinematics [15].

This write-up reports on recent developments and updates, in particular the possibility to use KaTie for calculations in Deep Inelastic Scattering (DIS).

\section{Updates}

KaTie can be obtained from

http://bitbucket.org/hameren/katie/downloads

Installation is rather straightforward, and is described, together with the further steps to be taken in order to arrive at event files, in the manual included in the package. Only a few more updates are reported here.

KaTie generates parton-level event files in the LHEF format [16] or a custom format, that can be used to obtain parton-level distributions of observables, or can be fed to a parton shower 
program. There is also the possibility to use KaTie itself to create histogram files for the desired observables. It takes three steps to arrive at the event files:

1. generation and compilation of source files specific to the requested scattering process. This takes very little time.

2. optimization of the phase space. This takes more time.

3. generation of the event files. This takes most time.

No actual timing numbers are given above because it completely depends on $e$.g. how many finalstate jets are requested. Several event files can be created in parallel by running an executable with different input seeds of the random number generator. These are then merged into an single file for which any set of events can be used for an unbiased estimate of the desired observables. One update is that this merging procedure can now be performed by a small FORTRAN program, for which a template is provided, and which can be adapted by the user to accommodate reweighting, and can already produce histograms of arbitrary distributions.

A single input file contains all information regarding the desired process. It must include a list of partonic sub processes the user wants to be include, e.g. two possible processes for dijets are

process $=g$ g $\rightarrow$ g $g$

process $=g \mathrm{~g} \rightarrow \mathrm{u}$ u

etc. The format has been simplified, and now options departing from defaults can be included separated by commas, e.g.

process $=g \mathrm{~g} \rightarrow \mathrm{u} u \sim$, factor $=\mathrm{Nf}$

The default factor is 1 . The desired powers of couplings in case of electro-weak interactions can now be included this way, or set for all processes e.g. with a line

pNonQCD $=200$

For the study in [14] it was essential to have the possibility for different sets of transverse moment dependent PDFs (TMDs) for each of the scattering hadrons. This is achieved with, for example

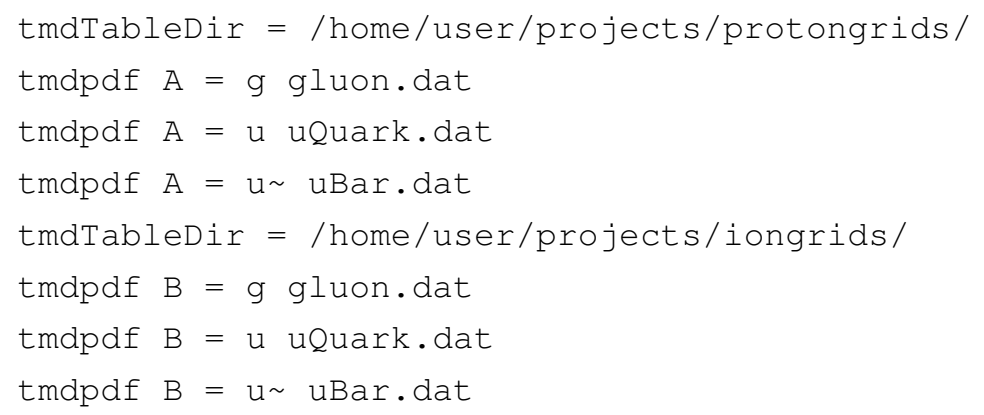

Here it was assumed that the TMD grid files are in different directories, but have the same name, which however is not necessary. The letter $\mathrm{A}$ refers to the positive-rapidity initial state, and the letter $B$ to the negative-rapidity initial state. In the process list, they refer to partons as B A $\rightarrow 1$ 23 etc. So the first one is B and the second one A.

It is now possible to include more complicated cuts and weight factors by providing pseudo FORTRAN source code, like 


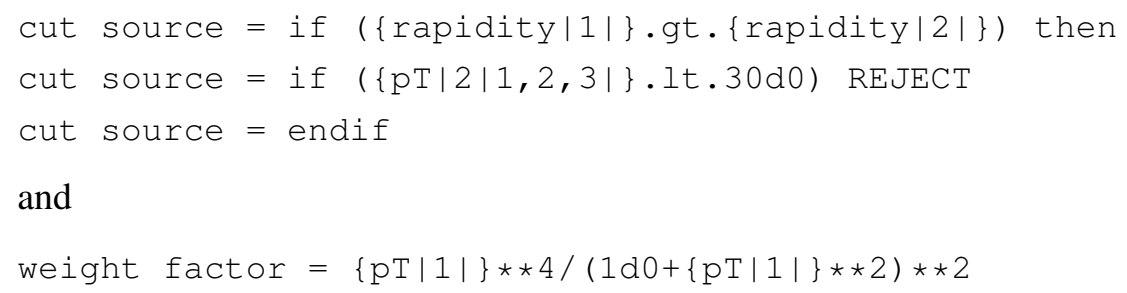

Also, now it is possible to set a separate renormalization scale, and separate factorization scales for each initial-state side e.g.

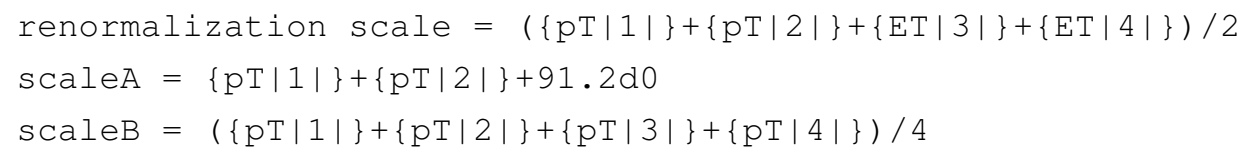

One major update is the possibility to create event files relevant for DIS. Partonic sub processes must be set as, for dijets for example,

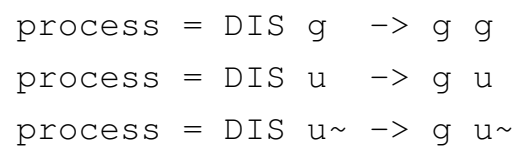

The, for example, first process line implies the process $e^{-} g-g \quad g e^{-}$, so the initial-state electron has negative rapidity. The user should not forget to set the proper number of electro-weak interactions, so at least

PNONQCD $=2 \quad 0 \quad 0$

The user can set beam energies EbeamPosRap and EbeamNegRap, but also the equivalents EbeamA and EbeamB, or EbeamHadron and EbeamElectron. This is also possible processes other than DIS. In order to set cuts involving the final-state electron, it is indicated by the word lepton (or electron or positron), for example

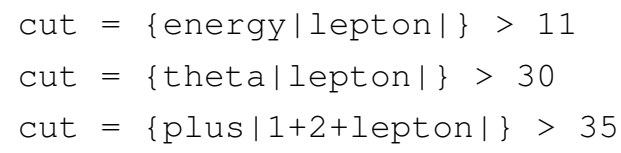

In this example, the kinematical variable plus is introduced, refering to the value of $E-p_{z}$ of the momentum represented by the argument, that is the Lorentz-invariant product of this momentum with $n_{+}^{\mu}=(1,0,0,1)$. Of course, also minus is available. Other available variables useful for DIS are $\{$ Qsquare $\}=Q^{2}$, $\{$ inelast $\}=y$, and $\{$ xBjorken $\}=x_{\mathrm{B}}$, satisfying $x_{\mathrm{B}} y=Q^{2} / E_{\mathrm{cm}}^{2}$. These three variables do not take arguments. Furthermore, there are deltaRbreit and pTbreit, referring to $\Delta R$ and $p_{T}$ in the Breit-frame of the difference between the momenta of the initial-state electron and the final-state electron. For $e^{+} p$ scattering, the keyword is DIS+, while DIS- is equivalent to DIS.

\section{A possible application for DIS}

Recently, forward-forward dijet correlation data measured at ATLAS for proton-proton and proton-lead collisions [17] were studied in the context of, among other things, gluon saturation [18]. 
The calculations were performed within the so-called Improved Transverse Momentum factorization [19], but one essential ingredient, namely a momentum inbalance for the final-state jets, is already present in lowest order hybrid high-energy factorization [20]. The momentum inbalance is necessary to have a non-trivial distribution for the angle between the jets, and its shape close to the back-to-back configuration is sensitive to saturation. It is also essential for the low- $x$ parton to be a gluon. This situation can also be achieved for dijets at electron-hadron colliders.
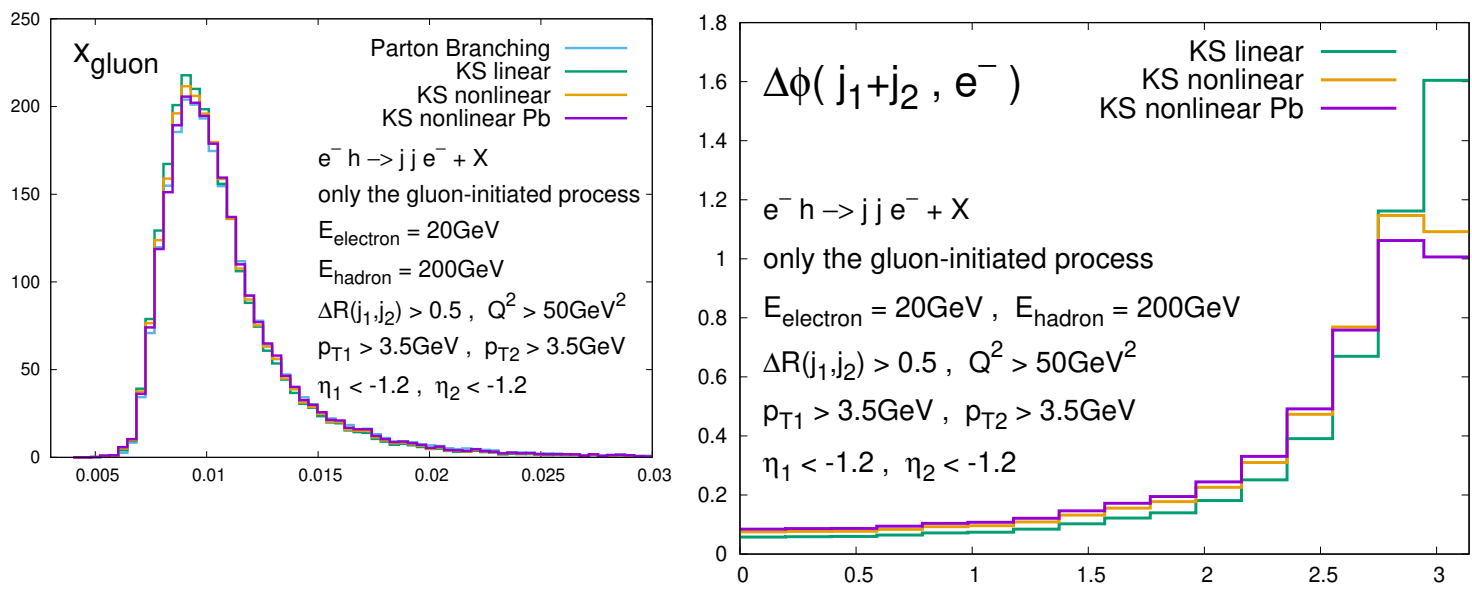

Figure 1: On the right is the normalized distribution of the angle between the final-state electron and the two jets for dijets in DIS at EIC-like kinematics. The left plot shows the normalized distribution of the energy fraction $x$ passed from the hadron to the initial state gluon. In this plot, the Parton Branching TMD [21] is included, because it is valid over the whole phase space and ensures that the cuts are appropriate. The different colors for the other TMDs correspond to different rates of saturation, included via different strengths of the non-linearity in the evolution.

Figure 1 shows results of calculations at EIC-like kinematics. The relevant observable sensitive to the final-state momentum inbalance, and therefore potentially to saturation, is the angle between the final-state electron and the two jets (this observable has been studied in other contexts in e.g. [22,23]). One can observe that different rates of saturation included in the Kutak-Sapeta gluon distributions [24] lead to distinguishable shapes for the angle distribution.

\section{References}

[1] A. Kanaki and C. G. Papadopoulos, HELAC: A Package to compute electroweak helicity amplitudes, Comput.Phys.Commun. 132 (2000) 306-315, [hep-ph/ 0002082 ].

[2] F. Krauss, R. Kuhn, and G. Soff, AMEGIC++ 1.0: A Matrix element generator in C++, JHEP 0202 (2002) 044, [hep-ph/ 0109036$].$

[3] M. Moretti, T. Ohl, and J. Reuter, O’Mega: An Optimizing matrix element generator, hep-ph/0102195.

[4] M. L. Mangano, M. Moretti, F. Piccinini, R. Pittau, and A. D. Polosa, ALPGEN, a generator for hard multiparton processes in hadronic collisions, JHEP 0307 (2003) 001, [hep-ph / 0206293 ]. 
[5] F. Maltoni and T. Stelzer, MadEvent: Automatic event generation with MadGraph, JHEP 0302 (2003) 027, [hep-ph/0208156].

[6] S. Catani, M. Ciafaloni, and F. Hautmann, High-energy factorization and small $x$ heavy flavor production, Nucl.Phys. B366 (1991) 135-188.

[7] J. C. Collins and R. K. Ellis, Heavy quark production in very high-energy hadron collisions, Nucl.Phys. B360 (1991) 3-30.

[8] A. van Hameren, KaTie : For parton-level event generation with $k_{T}$-dependent initial states, Comput. Phys. Commun. 224 (2018) 371-380, [1611.00680].

[9] A. van Hameren, P. Kotko, and K. Kutak, Helicity amplitudes for high-energy scattering, JHEP 1301 (2013) 078, [1211.0961].

[10] A. van Hameren, A note on QED gauge invariance of off-shell amplitudes, 1902.01791.

[11] M. Nefedov and V. Saleev, Off-shell initial state effects, gauge invariance and angular distributions in the Drell-Yan process, Phys. Lett. B790 (2019) 551-556, [1810 . 04061$].$

[12] M. Bury, H. Van Haevermaet, A. Van Hameren, P. Van Mechelen, K. Kutak, and M. Serino, Single inclusive jet production and the nuclear modification ratio at very forward rapidity in proton-lead collisions with $\sqrt{s_{N N}}=5.02$ TeV, Phys. Lett. B780 (2018) 185-190, [1712.08105].

[13] M. Deak, A. van Hameren, H. Jung, A. Kusina, K. Kutak, and M. Serino, Calculation of the Z+jet cross section including transverse momenta of initial partons, Phys. Rev. D99 (2019), no. 9094011, [1809.03854].

[14] E. Blanco, A. van Hameren, H. Jung, A. Kusina, and K. Kutak, Z boson production in proton-lead collisions at the LHC accounting for transverse momenta of initial partons, 1905.07331.

[15] M. Bury, A. van Hameren, H. Jung, K. Kutak, S. Sapeta, and M. Serino, Calculations with off-shell matrix elements, TMD parton densities and TMD parton showers, Eur. Phys. J. C78 (2018), no. 2 137, [1712.05932].

[16] J. Alwall et al., A Standard format for Les Houches event files, Comput. Phys. Commun. 176 (2007) 300-304, [hep-ph/0609017].

[17] ATLAS Collaboration, M. Aaboud et al., Dijet azimuthal correlations and conditional yields in pp and $p+P b$ collisions at $\sqrt{s_{\mathrm{NN}}}=5.02 \mathrm{TeV}$ with the ATLAS detector, 1901.10440 .

[18] A. van Hameren, P. Kotko, K. Kutak, and S. Sapeta, Broadening and saturation effects in dijet azimuthal correlations in $p$-p and p-Pb collisions at $\sqrt{\mathbf{S}_{\mathrm{NN}}}=5.02 \mathrm{TeV}, 1903.01361$.

[19] P. Kotko, K. Kutak, C. Marquet, E. Petreska, S. Sapeta, and A. van Hameren, Improved TMD factorization for forward dijet production in dilute-dense hadronic collisions, JHEP 09 (2015) 106, [1503.03421].

[20] A. Dumitru, A. Hayashigaki, and J. Jalilian-Marian, The Color glass condensate and hadron production in the forward region, Nucl. Phys. A765 (2006) 464-482, [hep-ph/ 0506308 ].

[21] F. Hautmann, H. Jung, A. Lelek, V. Radescu, and R. Zlebcik, Collinear and TMD Quark and Gluon Densities from Parton Branching Solution of QCD Evolution Equations, JHEP 01 (2018) 070, [1708.03279].

[22] C. Jacobsson, Jet Azimuthal Angle Asymmetries in Deep Inelastic Scattering as a test of QCD. PhD thesis, Lund University, 1994.

[23] H1 Collaboration, F. D. Aaron et al., Measurement of the Azimuthal Correlation between the most Forward Jet and the Scattered Positron in Deep-Inelastic Scattering at HERA, Eur. Phys. J. C72 (2012) 1910, [1111.4227].

[24] K. Kutak and S. Sapeta, Gluon saturation in dijet production in p-Pb collisions at Large Hadron Collider, Phys.Rev. D86 (2012) 094043, [1205. 5035$].$ 